SLAC-PUB-15914

\title{
Recent BABAR Charm physics results
}

\author{
Maurizio Martinelli*i \\ On behalf of the BABAR Collaboration. \\ Università degli Studi di Bari and INFN, Bari, 70124, ITALY \\ SLAC National Accelerator Laboratory, Stanford, California 94309 USA \\ E-mail: M.Martinellienikhef.nl
}

We report recent $B A B A R$ charm physics results, including $C P$ violation studies in $D^{+} \rightarrow K_{S}^{0} \pi^{+}$ and $D_{(s)}^{+} \rightarrow K^{+} K_{S}^{0} \pi^{+} \pi^{-}$, a study of the Dalitz plot of $D_{s}^{+} \rightarrow K^{+} K^{-} \pi^{+}$and the measurements of the mass and width of the $D_{s 1}(2535)^{+}$.

The 2011 Europhysics Conference on High Energy Physics, EPS-HEP 2011,

July 21-27, 2011

Grenoble, Rhône-Alpes, France

\footnotetext{
*Speaker.

${ }^{\dagger}$ now at NIKHEF, Science Park 105, 1098 XG Amsterdam, The Netherlands.
} 
The BABAR experiment has a broad Charm physics program and has published several new results recently. Among them, four main topics can be identified: precision measurements, Dalitz plot analysis, $C P$ violation and rare decays. In the following, the most recent results will be briefly outlined, except for those concerning rare decays.

\section{Precision measurements}

The mass and width of the $D_{s 1}(2535)^{+}$[1] meson have been recently measured with high precision by the BABAR experiment [2]. The study of $D_{s j}$ mesons is important, since their masses and widths are not always in agreement with potential model calculations [3]. There are many alternative theoretical explanations, such as $D^{*} K$ molecules, chiral partners, unitarized chiral model, tetraquarks and lattice calculations $[4,5]$. The precise measurement of the mass and width, and the study of the spin-parity of the $D_{s 1}(2535)^{+}$may lead to a better understanding of the $D_{s j}$ meson framework.

The $D_{s 1}(2535)^{+}$meson is reconstructed in the decay mode $D^{*+} K_{S}^{0}$, where $K_{S}^{0} \rightarrow \pi^{+} \pi^{-}$and $D^{*+} \rightarrow D^{0} \pi^{+}$. Two Cabibbo-favored $D^{0}$ decay modes are considered: $D^{0} \rightarrow K^{-} \pi^{+}$and $D^{0}$ $\rightarrow K^{-} \pi^{+} \pi^{+} \pi^{-}$. The signal region is defined by a combined selection on the $\mathrm{m}\left(D^{0}\right), \mathrm{m}\left(D^{*+}\right)$ $\mathrm{m}\left(D^{0}\right)$ and $\mathrm{m}\left(K_{S}^{0}\right)$ mass spectra. The distribution of $\Delta m=\mathrm{m}\left(D_{s 1}(2535)^{+}\right)-\mathrm{m}\left(D^{*+}\right)$ for the signal region is fitted to a relativistic Breit-Wigner convolved with the resolution function obtained from Monte Carlo simulation. The results of the fit are: $\mathrm{m}\left(D_{s 1}(2535)^{+}\right)=2535.08 \pm 0.01 \pm 0.15 \mathrm{MeV} / c^{2}$ and $\Gamma\left(D_{s 1}(2535)^{+}\right)=0.92 \pm 0.03 \pm 0.04 \mathrm{MeV}$, where the first error is statistical and the second is systematic. The main systematic uncertainty on the mass measurement is due to the reconstruction of the tracks in the detector, while the measurement of the width is mainly affected by the resolution model. The systematic uncertainty associated with the fit procedure is found to be small.

An angular analysis has been performed on signal events. The distributions of the cosines of the $D^{0}$ and $D^{*+}$ helicity angles were used, and were found to be consistent with the $J^{P}=1^{+}$ assignment.

\section{Dalitz plot analysis}

A Dalitz plot analysis of $D_{s}^{+} \rightarrow K^{+} K^{-} \pi^{+}$has been published recently [6]. One motivation for this analysis is that the scalar mesons are still a puzzle in light meson spectroscopy. The question is whether the $a_{0}(980)$ and $f_{0}(980)$ are 4-quark states due to their proximity to $K^{+} K^{-}$threshold [7]. Furthermore, the measurement of $K^{+} K^{-} S$-wave amplitude near threshold is of great importance for the precise measurement of $C P$ violation in $B_{S}$ oscillations using $B_{S} \rightarrow J / \psi \phi[8,9]$.

In this analysis, an high purity sample of $100,000 D_{s}^{+} \rightarrow K^{+} K^{-} \pi^{+}$events has been selected from $D_{s}^{*}(2112)^{+} \rightarrow D_{s}^{+} \gamma$ decays using a selection on likelihood ratio and on $\mathrm{m}\left(K^{+} K^{-} \pi^{+} \gamma\right)-\mathrm{m}\left(K^{+}\right.$ $K^{-} \pi^{+}$). Initially, a model-independent representation of the $K^{+} K^{-} S$-wave amplitude is extracted from the projections of the first three Legendre polynomial moments in the $\phi$-meson mass region. An adaptive parametrization of the $S$-wave is then included into the Dalitz plot model to describe the $f_{0}(980)$. The model consists of the coherent superposition of six quasi-two-body resonance contributions (in order of measured decay fraction): $\bar{K}^{*}(892)^{0}, \phi(1020), f_{0}(980), \bar{K}^{*}(1430)^{0}, f_{0}(1710)$, 
$f_{0}(1370)$. The total decay fraction is $110.2 \pm 0.6 \pm 2.0 \%$, and the goodness of fit, tested using adaptive binning, gives $\chi^{2} / N D F=2843 /(2305-14)=1.24$.

Compared to previous analyses from E687 [10] (700 events) and CLEO-c [11] (12,000 events), the use of a model-independent parametrization overcomes the uncertainties introduced by the coupling constants in a Flatté description of the $S$-wave amplitude, and gives a more precise description of the Dalitz plot and the corresponding fit fractions.

\section{3. $C P$ violation}

The large samples of charm meson decays recorded at the $B$ factories and at hadron collider experiments allow the performance of precise tests of $C P$ violation. The sensitivity of these measurements has indeed reached the higher Standard Model (SM) expectations of $10^{-3}$. Furthermore, there are alternative models in which New Physics can introduce processes that generate $C P$ violation to the level of $1 \%$ at most. Given the smaller values expected from the SM, any measurement greater than $10^{-3}$ would be a clear indication of processes beyond the SM.

\subsection{Direct $C P$ violation}

The most intuitive way to search for $C P$ violation is to measure the asymmetry between the $D$ and $\bar{D}$ decay rates. However, at the asymmetric detectors, like $B A B A R$, the forward-backward asymmetry $\left(A_{F B}\right)$ effect to the detector geometry needs to be taken into account, as well as the asymmetry introduced by the different detection efficiency between particle and anti-particle $\left(A_{\varepsilon}\right)$. The asymmetry between the $D$ and $\bar{D}$ decay rates is then $A=\frac{\Gamma_{D}-\Gamma_{\bar{D}}}{\Gamma_{D}+\Gamma_{\bar{D}}}=A_{C P}+A_{F B}+A_{\varepsilon}$.

A search for $C P$ violation in $D^{+} \rightarrow K_{S}^{0} \pi^{+}$decays has been published recently by BABAR [12]. In this analysis, the particle-anti-particle asymmetry $A_{\varepsilon}$ has been measured using a control sample of pions from $B \bar{B}$ decays, and has been removed from the data sample weighting the $D^{-}$candidates. The forward-backward and $C P$ violation asymmetry have been disentangled by making use of the fact that $A_{F B}$ has an odd dependence on $\cos \theta_{D}^{*}$, the cosine of the $D$ production angle in the centerof-mass frame, while $A_{C P}$ is even. These can then be measured using $A_{C P}=1 / 2\left[A\left(\left|\cos \theta_{D}^{*}\right|\right)+\right.$ $\left.A\left(-\left|\cos \theta_{D}^{*}\right|\right)\right]$ and $A_{F B}=1 / 2\left[A\left(\left|\cos \theta_{D}^{*}\right|\right)-A\left(-\left|\cos \theta_{D}^{*}\right|\right)\right]$.

Averaging the $C P$ violation measurements in the five ranges of $\left|\cos \theta_{D}^{*}\right|$ considered, the final result is $A_{C P}=(-0.44 \pm 0.13 \pm 0.10) \%$.

\section{2 $C P$ violation using $T$-odd correlations}

The violation of $C P$ can be searched for indirectly by using $T$-odd correlations. Observing a $T$-odd correlation, $T$ violation can be claimed, and $C P$ violation then results from the assumption of the validity of the $C P T$ theorem.

A $T$-odd observable can be built using the momenta of the daughter particles in the rest frame of the decaying parent meson. For example, $C_{T}=\vec{p}_{K^{+}} \cdot\left(\vec{p}_{\pi^{+}} \times \vec{p}_{\pi^{-}}\right)$, in the case of $D_{(s)}^{+} \rightarrow K^{+} K_{S}^{0}$ $\pi^{+} \pi^{-}$decay, studied recently by BABAR [13]. The asymmetry of $D$ decays with $C_{T}$ greater or less than $0, A_{T}$, would indicate $T$-odd correlations, but the effect of final state interactions could fake this result by introducing a strong phase in the asymmetry. In order to remove the strong phase, the charge-conjugate of $A_{T}, \bar{A}_{T}$, can be measured for $\bar{D}$ decays and a $T$ violating asymmetry could then appear in $\mathscr{A}_{T}=1 / 2\left(A_{T}-\bar{A}_{T}\right)$. 
The $D_{(s)}^{+} \rightarrow K^{+} K_{S}^{0} \pi^{+} \pi^{-}$events are selected by means of a likelihood ratio criterion which yields 20,000 and 30,000 signal events for $D^{+}$and $D_{s}^{+}$, respectively. The dataset is then divided into four sub-samples, depending on $D$ charge and $C_{T}\left(\bar{C}_{T}\right)$ value, both for $D^{+}$and $D_{s}^{+}$. The sub-samples are simultaneously fit to a model of two Gaussians with common mean and a linear (second order polynomial) $D^{+}\left(D_{s}^{+}\right)$background. The asymmetries are obtained from the fit, and the results are: $A_{T}\left(D^{+}\right)=(+11.2 \pm 14.1 \pm 5.7) \times 10^{-3}, A_{T}\left(D^{-}\right)=(+35.1 \pm 14.3 \pm 7.2) \times 10^{-3}$, $A_{T}\left(D_{s}^{+}\right)=(-99.2 \pm 10.7 \pm 8.3) \times 10^{-3}, A_{T}\left(D_{s}^{-}\right)=(-72.1 \pm 10.9 \pm 10.7) \times 10^{-3}$. The effect of final state interaction is larger in $D_{s}^{+}$and $D^{0}$ decays [14] than in $D^{+}$decays. This result has been discussed in [15].

Combining the results to measure the $C P$ violation parameters, $\mathscr{A}_{T}\left(D^{+}\right)=(-12.0 \pm 10.0 \pm$ 4.6) $\times 10^{-3}, \mathscr{A}_{T}\left(D_{s}^{+}\right)=(-13.6 \pm 7.7 \pm 3.4) \times 10^{-3}$, so that the observables are consistent with zero, and indicate no effects of physics beyond the SM.

\section{Conclusions}

The BABAR Collaboration is still producing excellent Charm physics results. The large BABAR sample of Charm meson decays allows us to perform precision measurements and detailed Dalitz plot analyses. This dataset can also be used to search for $C P$ violation, obtaining sensitivities down to $10^{-3}$, but as yet there is no evidence of $C P$ violation in Charm decays, neither from SM, nor from beyond-SM, processes. Despite the end of data-taking in 2008, there are still many analyses in the pipeline, and other quality results are expected soon.

\section{References}

[1] The use of charge conjugate reactions is implied throughout, unless indicated otherwise.

[2] J. P. Lees et al. [ The BABAR Collaboration ], Phys. Rev. D83, 072003 (2011).

[3] R. N. Cahn, J. D. Jackson, Phys. Rev. D68, 037502 (2003).

[4] P. Colangelo, F. De Fazio, R. Ferrandes, Mod. Phys. Lett. A19 (2004) 2083-2102.

[5] E. S. Swanson, Phys. Rept. 429 (2006) 243-305.

[6] P. del Amo Sanchez et al. [ The BABAR Collaboration ], Phys. Rev. D83 (2011) 052001.

[7] F. E. Close, N. A. Tornqvist, J. Phys. G G28 (2002) R249-R267.

[8] S. Stone, L. Zhang, Phys. Rev. D79 (2009) 074024.

[9] Y. Xie, P. Clarke, G. Cowan, F. Muheim, JHEP 0909 (2009) 074.

[10] P. L. Frabetti et al. [ E687 Collaboration ], Phys. Lett. B351 (1995) 591-600.

[11] R. E. Mitchell et al. [ CLEO Collaboration ], Phys. Rev. D79 (2009) 072008.

[12] P. del Amo Sanchez et al. [ The BABAR Collaboration ], Phys. Rev. D83 (2011) 071103.

[13] J. P. Lees et al. [ The BABAR Collaboration ], Phys. Rev. D84 (2011) 031103.

[14] P. del Amo Sanchez et al. [ The BABAR Collaboration ], Phys. Rev. D81 (2010) 111103.

[15] M. Gronau, J. L. Rosner, [arXiv:1107.1232 [hep-ph]]. 